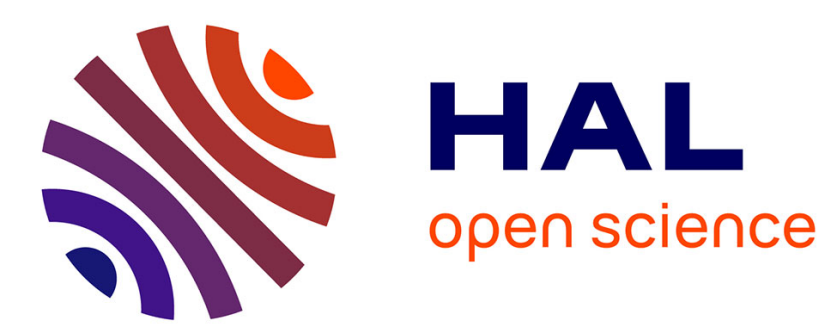

\title{
Thermal weakening of cracks and brittle-ductile transition of matter: A phase model
}

Tom Vincent-Dospital, Renaud Toussaint, Alain Cochard, Knut Jørgen

Måløy, Eirik Flekkøy

\section{- To cite this version:}

Tom Vincent-Dospital, Renaud Toussaint, Alain Cochard, Knut Jørgen Måløy, Eirik Flekkøy. Thermal weakening of cracks and brittle-ductile transition of matter: A phase model. Physical Review Materials, 2020, 4 (2), 10.1103/PhysRevMaterials.4.023604 . hal-03014256

\section{HAL Id: hal-03014256 \\ https://hal.science/hal-03014256}

Submitted on 19 Nov 2020

HAL is a multi-disciplinary open access archive for the deposit and dissemination of scientific research documents, whether they are published or not. The documents may come from teaching and research institutions in France or abroad, or from public or private research centers.
L'archive ouverte pluridisciplinaire HAL, est destinée au dépôt et à la diffusion de documents scientifiques de niveau recherche, publiés ou non, émanant des établissements d'enseignement et de recherche français ou étrangers, des laboratoires publics ou privés. 


\title{
Thermal weakening of cracks and brittle-ductile transition of matter: A phase model
}

\author{
Tom Vincent-Dospital $\odot,{ }^{1,2, *}$ Renaud Toussaint $\odot,{ }^{1,2, \dagger}$ Alain Cochard $\odot,{ }^{1}$ Knut Jørgen Måløy $\odot,{ }^{2}$ and Eirik G. Flekkøy ${ }^{2}$ \\ ${ }^{1}$ Université de Strasbourg, CNRS, Institut de Physique du Globe de Strasbourg, UMR 7516, \\ 5 rue René Descartes, F-67000 Strasbourg, France \\ ${ }^{2}$ SFF Porelab, The Njord Centre, Department of Physics, University of Oslo, P. O. Box 1048, Blindern, N-0316 Oslo, Norway
}

(Received 14 December 2018; revised manuscript received 19 June 2019; accepted 15 January 2020; published 24 February 2020)

\begin{abstract}
We present a model for the thermally activated propagation of cracks in elastic matrices. The propagation is considered as a subcritical phenomenon, the kinetics of which is described by an Arrhenius law. In this law, we take the thermal evolution of the crack front into account, assuming that a portion of the released mechanical energy is transformed into heat in a zone surrounding the tip. We show that such a model leads to a two-phase crack propagation: the first phase at low velocity in which the temperature elevation is of little effect and the propagation is mainly governed by the mechanical load and by the toughness of the medium, and the second phase in which the crack is thermally weakened and propagates at greater velocity. We illustrate, with numerical simulations of mode I cracks propagating in thin disordered media, how such a dual behavior can explain the usual stick-slip in brittle fracturing. In addition, we predict the existence of a limiting ambient temperature above which the weakened phase ceases to exist and we propose this critical phenomenon as a novel explanation for the brittle-ductile transition of solids.
\end{abstract}

DOI: 10.1103/PhysRevMaterials.4.023604

\section{INTRODUCTION}

Of paramount importance in engineering and geophysics, the impact of temperature in fracturing processes has since long been studied. It can simplistically be sorted into two categories: background effects where the temperature is treated as an environmental constant affecting the rates at which the defects of a medium are propagating or healing [1-4] and dynamic effects where the propagation of fractures selfinduces a rise in temperature in the vicinity of the crack front [5-9]. In the latter case, the heat elevation can be regarded as more than a secondary effect of the medium's damage: it can be an active process back affecting the crack propagation. This phenomenon will be here referred to as "thermal weakening." Such a weakening has notably been studied in earth science where it is believed to play a role in faults stability and earthquake triggering $[10,11]$ and it was included in the so-called rate-and-state framework [12] as an explanation for rate weakening faults. Several mechanisms have been proposed to explain thermal weakening, such as the softening $[13,14]$ or melting of fracture surfaces or the thermopressurization of fault fluids [15-17]. We here consider a model which disregards such effects and focuses on the statistical physics consideration of higher reaction rates (i.e., quicker fracture propagation) at higher temperatures, as implied by an Arrhenius law [18]. This model notably showed good agreement with the rupture dynamics, experimentally reported in various polymers [19]. In this work, we further discuss how, in addition, it stands as a physical explanation for the brittle-ductile transition of matter.

\footnotetext{
*vincentdospitalt@unistra.fr

†renaud.toussaint@unistra.fr
}

\section{THE THERMAL WEAKENING MODEL}

Arrhenius based models for the velocity of crack fronts have long been considered $[1,2,4,20]$ and have recently been shown to show good agreement with experimental observables of mode I cracks slowly propagating in acrylic glass bodies [21-23]. The rupture is then not considered as a Griffith-like threshold mechanism [24] where the crack only advances for $G>G_{c}$, where $G$ is the energy release rate of the crack in $\mathrm{J} \mathrm{m}^{-2}$ (arising from the mechanical load given to the crack front) and $G_{c}$ the fracture energy of the medium (the energy barrier per surface unit to overcome molecular bonds). It is rather considered as a thermally activated subcritical phenomenon $\left(G<G_{c}\right)$ for which the crack velocity is expressed as

$$
V=\alpha \nu \exp \left(\frac{\alpha^{2}\left(G-G_{c}\right)}{k_{B} T}\right),
$$

where $\alpha$ is a characteristic size (m) of the fracturing process, that is associated with its energy barrier. $k_{B} \approx 1.38 \times 10^{-23}$ $\mathrm{J} \mathrm{K}^{-1}$ is the Boltzmann constant, $T$ the absolute temperature at the crack tip and $v$ the thermal bath collisional frequency. Equation (1), as any Arrhenius law, is a continuous expression of a discrete process arising at the molecular scale. Cochard et al. [23] have recently discussed it at length. The exponential term is the probability (i.e., $<1)$ for the thermal agitation to exceed the activation energy $-\alpha^{2}\left(G-G_{c}\right)$ and hence for the crack to advance by a length $\alpha$. This probability is challenged every $1 / v$ seconds. In theory $v$ is also temperature dependent but this is of negligible effect compared to the exponential dependence of the probability term [18] and we hence define $V_{0}=\alpha \nu$, the maximum crack velocity obtained when the activation energy is always reached. $V_{0}$ shall typically be in the range of the Rayleigh surface wave velocity [25]. Because we consider the thermal evolution around the crack tip we also 


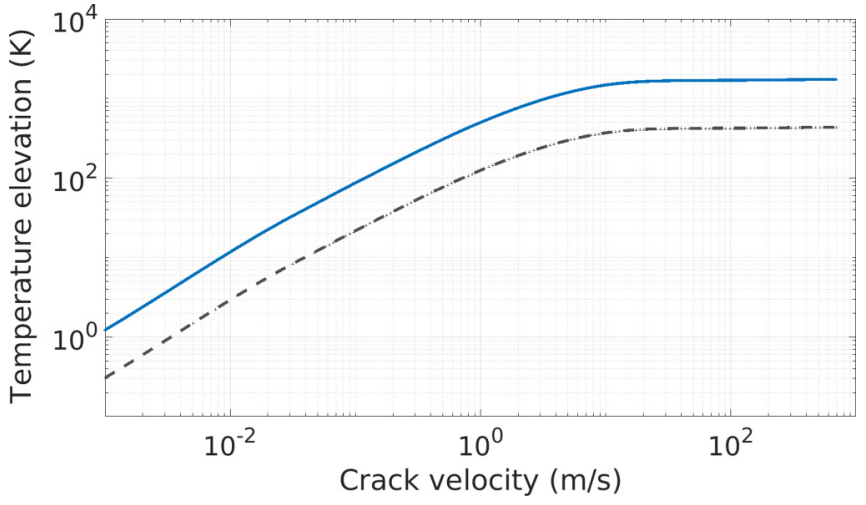

FIG. 1. Steady-state values of the temperature elevation. It is obtained by solving Eq. (2) for a crack propagating at a constant velocity and for $\phi G=200$ (plain plot) and $50 \mathrm{~J} \mathrm{~m}^{-2}$ (dotted plot).

note $T=T_{0}+\Delta T$, where $T_{0}$ is the ambient temperature and $\Delta T$ any variation away from it at the tip.

Such variations are induced by the dissipation of the mechanical energy given to the elastic matrix in a plastic zone that surrounds the crack tip [26]. There are many processes responsible for such an energy loss, as the creation of new defects surfaces and the emission of mechanical waves, but we here focus on the release of heat. The model we use is based on the work of Toussaint et al. [9]: a portion $\phi$ of the energy release rate is dissipated on a cylindrical zone of radius $l$ centered around the crack tip. Such a configuration leads to a thermal evolution governed by

$$
\frac{\partial(\Delta T)}{\partial t}=\frac{\lambda}{C} \nabla^{2}(\Delta T)+\frac{\phi G V}{C \pi l^{2}} f,
$$

which is a diffusion equation including a source term. $\lambda$ is the medium's thermal conductivity in $\mathrm{J} \mathrm{s}^{-1} \mathrm{~m}^{-1} \mathrm{~K}^{-1}, C$ is the volumetric heat capacity in $\mathrm{J} \mathrm{K}^{-1} \mathrm{~m}^{-3}, t$ is the time variable and $\nabla^{2}$ is the Laplace operator. $f$ is the support function of the heat production zone of surface integral $\pi l^{2}$ (i.e., $f=1$ in the zone and $f=0$ otherwise). Solving this equation for a crack propagating at a constant velocity and constant release rate, one can show that the thermal elevation at the tip reaches a steady state after a short transient time. Figure 1 shows the evolution of this steady state as a function of $V$ and for two values of $G$. See Ref. [27] for details on its computation. In our model, we use this relation to describe $\Delta T(V, G)$, thus discarding any transient regime. Equation (1) becomes

$$
V=V_{0} \exp \left(\frac{\alpha^{2}\left(G-G_{c}\right)}{k_{B}\left[T_{0}+\Delta T(V, G)\right]}\right) .
$$

\section{Parameters used for illustration}

Note that most of the previously introduced parameters are strongly dependent on the medium in which the crack propagates. The figures we display here use parameters that could be likely for the propagation of interfacial cracks in sintered acrylic glass bodies [21,22] and are discussed in the Supplemental Material Ref. [27]: $\alpha=2.5 \times 10^{-11} \mathrm{~m}, G_{c}=$ $250 \mathrm{~J} \mathrm{~m}^{-2}, T_{0}=293 \mathrm{~K}, C=1.7 \times 10^{6} \mathrm{~J} \mathrm{~K}^{-1} \mathrm{~m}^{-3}, \lambda=0.19$ $\mathrm{J} \mathrm{s}^{-1} \mathrm{~m}^{-1} \mathrm{~K}^{-1}, V_{0}=1000 \mathrm{~m} \mathrm{~s}^{-1}, l=20 \mathrm{~nm}$, and $\phi=1$. Note that we use this set of values only to propose some likely

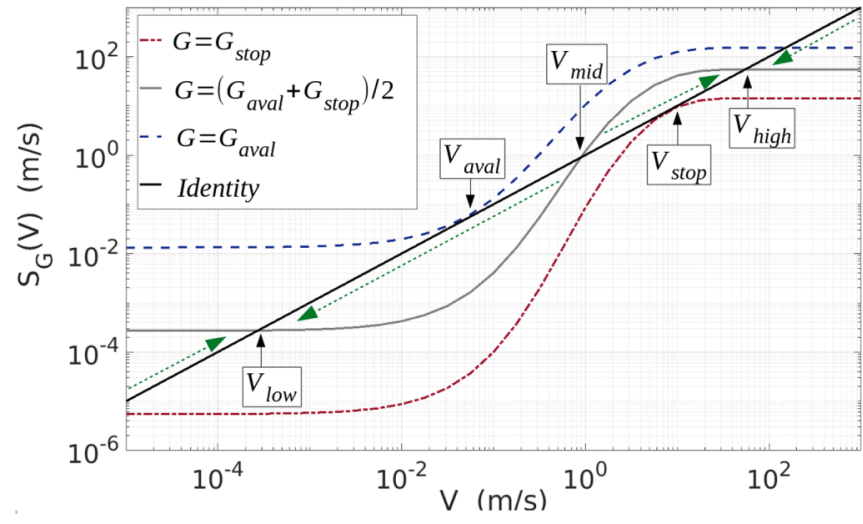

FIG. 2. Representation of $V=S_{G}(V)$ for three values of $G$ : $G_{\text {stop }}, G_{\text {aval }}\left(>G_{\text {stop }}\right)$ and the mid-value between $G_{\text {stop }}$ and $G_{\text {aval }}$. The intersections of $S_{G}$ with the identity plot (straight line) give the possible crack velocities. They are denoted $V_{\text {low }}, V_{\text {mid }}$, and $V_{\text {high }}$ and are emphasized for the intermediate $G$ plot. $V_{\text {aval }}$ and $V_{\text {stop }}$ are indicated on the two others plots. The dashed arrows indicate how off-balanced situations evolve to a stable fixed point.

orders of magnitude for our parameters, and not to accurately represent the rupture of a specific material, as done in [19].

\section{PHASE BEHAVIOR}

Equation (3) defines, for a given load $G$, a function $S_{G}$ such as $V=S_{G}(V)$. To fit the model, the actual velocity at which a crack advances must be a solution of this equation (i.e., be a fixed point for the function $S_{G}$ ) [28]. Figure 2 illustrates that, depending on the value of $G, S_{G}$ has one to three fixed points: three possible values for the crack velocity. This finite number of solutions arises from the steady-state approximation. If we were to consider the transient regimes, $S_{G}(V)$ would be, for a front propagating at any velocity $V$ and load $G$, a target velocity. Any crack not having reached a steady state would thus accelerate or slow down to follow this function. The intermediate fixed point, when it exists, is then unstable (virtually impossible): a crack with a velocity value just above this point $\left[V<S_{G}(V)\right]$ is too slow to be steady. The heat generation at the tip is higher than what the diffusion can accommodate, the temperature rises and the velocity increases to converge to the upper fixed point. On the contrary, if a crack is slightly slower than the intermediate solution $\left[V>S_{G}(V)\right]$, the crack cools down to the lower fixed point. We here assume that such transitions happen in a negligible time so the steady velocities are sufficient to describe the main dynamics. The outer solutions of (3) being the only stable ones, the model displays a two-phase behavior. The lower velocity marks a slow phase. The temperature elevation at the crack tip has little effect on the propagation, as $\Delta T(V, G) \ll T_{0}$. The higher solution corresponds to a thermally weakened phase where $\Delta T(V, G)$ has reached the plateau temperature of Fig. 1. The velocity is there increased as the induced heat is potentially significant compared to the thermal background.

Notice in Fig. 2 that there are two particular values of the load $G$ for which either the lower or the higher phase ceases to exist. We denote them $G_{\text {aval }}$ and $G_{\text {stop }}$ (with $G_{\text {aval }}>G_{\text {stop }}$ ) as they correspond to mechanical loads at which a slow crack 


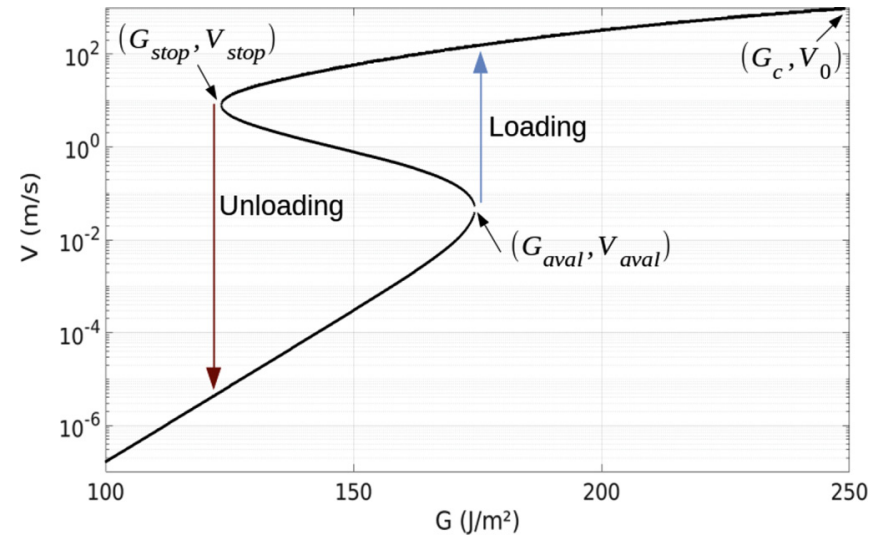

FIG. 3. Solutions for the crack velocity as a function of $G$ for $T_{0}=293 \mathrm{~K}$. All solutions in between $V_{\text {stop }}$ and $V_{\text {aval }}$ are unstable, any other point is a possible crack velocity. The arrows represent how a crack avalanches of slows down at the phase transition thresholds.

will have to avalanche to the thermally weakened phase or at which a fast (weakened) crack can only cool down to the slow phase. For $G$ in between these two thresholds, a hysteresis situation holds, there are several solutions for $V$ and the crack might or might not be thermally weakened, depending on the mechanical history. To $G_{\text {aval }}$ and $G_{\text {stop }}$ correspond some specific velocities $V_{\text {aval }}<V_{\text {stop }}$ in between which a crack cannot propagate, as any solution is there unstable. Figure 3 shows the possible crack velocities for various values of $G$. One can notice how similar it is to a first order phase transition [29] for the order parameter $V$ associated to avalanches (jumps in $V$ ) triggered by variations in the driving field $G$ at temperature $T_{0}$. Such a description compares interestingly with various $(V, G)$ branches that are experimentally reported, for instance in the rupture dynamics of pressure adhesives [30,31], PMMA [21,32], or elastomers [33] and the model can hence be matched to actual data over decades of velocities [19]. Note that, in the hysteresis domain, we do not discriminate on the relative stability of each phase. One can however argue, by analogy with other phase transition systems [29], that one of the two solutions could only be metastable, that is, in an equilibrium which is less energetically favorable than the one of the alternative phase. In this case, when traveling though an heterogeneous medium where the variations in fracture energy are enough to get shifts from only one state to the other, one of the phase could still be preferential for the crack propagation.

\section{CRITICAL POINT}

Besides $G, T_{0}$ is the only other parameter of (3) which is not dependent on the medium's properties. Figure 4 thus shows the predicted propagation velocities for various ambient temperatures. Notice the existence of a critical ambient temperature: $T_{0}^{*}$, at which $G_{\text {aval }}=G_{\text {stop }}=G^{*}$ and $V=V^{*}$. Beyond $T_{0}^{*}$, the Joule effect cannot overcome the thermal background enough for the crack to be weakened. Increasing the load then only leads to a smooth increase in the velocity. To relate to the theory of critical phenomena in phase transitions [29], we

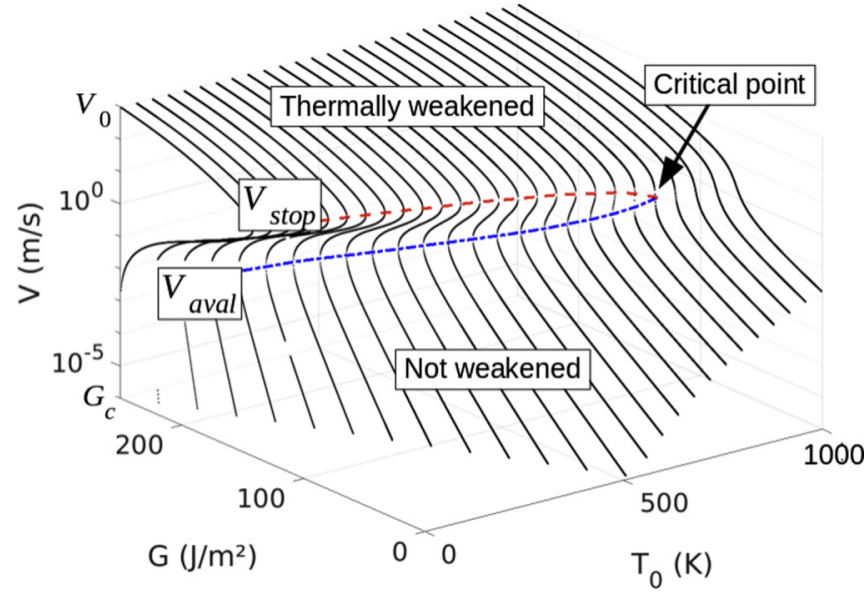

FIG. 4. Solutions for the crack velocity as a function of $G$ and for various $T_{0}$. The dashed lines show the $\left(V_{\text {stop }}, G_{\text {stop }}\right)$ and $\left(V_{\text {aval }}, G_{\text {aval }}\right)$ couples and converge to the critical point.

looked for the real numbers $\beta, \delta$, and $\gamma$ such that

$$
\begin{aligned}
& \frac{V-V^{*}}{V^{*}} \sim\left(\frac{T_{0}-T_{0}^{*}}{T_{0}^{*}}\right)_{G=G^{*}}^{\beta}, \frac{G-G^{*}}{G^{*}} \sim\left(\frac{V-V^{*}}{V^{*}}\right)_{T_{0}=T_{0}^{*}}^{\delta}, \\
& \frac{G^{*}}{V^{*}} \frac{\partial V}{\partial G} \sim\left(\frac{T_{0}-T_{0}^{*}}{T_{0}^{*}}\right)_{G=G^{*}}^{-\gamma},
\end{aligned}
$$

where $\sim$ stands for a mathematical equivalence in the vicinity of the critical point (any pre-factor is overlooked). These exponents describe how $V$ converges towards $V^{*}$ beyond the critical point $\left(T_{0} \geqslant T_{0}^{*}\right)$. We also characterized how the hysteresis domain shrinks, looking for $\beta^{\prime}, \delta^{\prime}$, and $\gamma^{\prime}$ such that

$$
\begin{gathered}
\frac{V_{\text {stop }}-V_{\text {aval }}}{V^{*}} \sim\left(\frac{T_{0}^{*}-T_{0}}{T_{0}^{*}}\right)^{\beta^{\prime}}, \\
\frac{G_{\text {aval }}-G_{\text {stop }}}{G^{*}} \sim\left(\frac{V_{\text {stop }}-V_{\text {aval }}}{V^{*}}\right)^{\delta^{\prime}}, \\
\frac{G^{*}}{V^{*}} \frac{V_{\text {stop }}-V_{\text {aval }}}{G_{\text {aval }}-G_{\text {stop }}} \sim\left(\frac{T_{0}^{*}-T_{0}}{T_{0}^{*}}\right)^{-\gamma^{\prime}} .
\end{gathered}
$$

With a bisection, we numerically estimated the critical point, checking for the number of solutions of $V=S_{G}(V)$ (three solutions below $T_{0}^{*}$ and one above). Analyzing the shape of the velocity map in the derived vicinity, we found: $\beta \approx$ $1 / 3, \delta \approx 3, \gamma \approx 2 / 3$, and $\beta^{\prime} \approx 1 / 2, \delta^{\prime} \approx 3, \gamma^{\prime} \approx 1$ (see Ref. [27]). Both sets of exponents respect the scaling relation [29]: $2 \beta+\gamma=\beta(\delta+1)$. We hence derived critical exponents which are, along the phase co-existence domain, the same as the mean field exponents for, say, the liquidgas transition [29], but different beyond the critical point. The mean-field characteristic might arise from the statistical nature of the Arrhenius law only representing an average velocity while consecutive molecular bonds can be overcome at very different speeds. Another interpretation is that it translates the zero-dimensional character of our model. We have indeed disregarded any velocity variations and elastic 


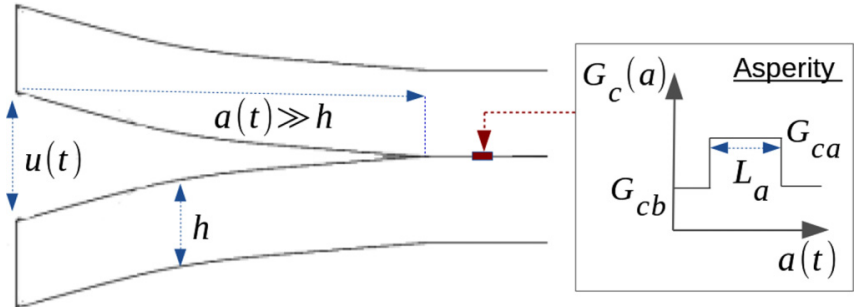

FIG. 5. Geometry for the numerical simulations of zerodimensional crack fronts overcoming a tough asperity.

interactions along the crack front, making the assumption that it is thin or symmetrical enough perpendicularly to the propagation direction.

\section{SIMULATIONS OF OD FRONTS IN DISORDERED MEDIA}

Let us finally illustrate the phase transitions with some simulations of such zero-dimensional fronts loaded in mode I. The loading geometry that we consider is shown in Fig. 5. The support body consists in two sintered elastic plates which are progressively separated at the edge. The deflection on the side, $u(t)$ (in $\mathrm{m}$ ), is increased linearly with time: $u(t)=v_{u} t$. Using the Euler-Bernoulli beam theory [34], one can compute the energy release rate at the tip of such a system:

$$
G(t)=\frac{3 E h^{3} v_{u}^{2} t^{2}}{8 a(t)^{4}} \quad \text { if } a \gg h,
$$

with $E$ the body Young modulus (in Pa), $h$ half of its thickness and $a$ the crack advancement such as $V=\partial a / \partial t$. By inserting (10) in (3), we obtain the differential equation in $a(t)$ that governs the crack progression and that we solved with a time step adaptive Runge-Kutta algorithm [35]. We here consider a crack interface with a homogeneous background cohesion $G_{c}=G_{c_{b}}$ which is only disturbed by a single tough asperity of length $L_{a}\left(G_{c_{a}}>G_{c_{b}}\right)$. Figure 5 shows a schematic for this anomaly while Fig. 6 shows, for several values of $G_{c_{a}}$, the course of the crack over it and the corresponding evolution of the energy release rate. When the front reaches the asperity, the crack velocity dramatically decreases as it reaches a tougher area. Meanwhile the load $G$ increases because the far field deflection continues to build up on a now quasistatic crack. Once the anomaly finally gets passed, the simulations show two possible scenarios. If $G_{\text {aval }}\left(G_{c_{b}}\right)$ (i.e., the phase shift threshold for the background $G_{c_{b}}$ ) was not reached over the anomaly, then the crack only accelerates back to its pre-asperity state. However, if $G_{\text {aval }}\left(G_{c_{b}}\right)$ was overcome, the crack shifts phase and becomes thermally weakened: it avalanches until $G=G_{\text {stop }}$. In Fig. 6, one can read the values of $G_{\text {aval }}$ and $G_{\text {stop }}$ and remark that they match the theoretical values displayed in Fig. 3. Note that, if the load was to be quickly increased, an avalanche could be triggered without the need for any asperity. We showed, however, how the medium's disorder can lead to some spontaneous thermal weakening of the crack course.

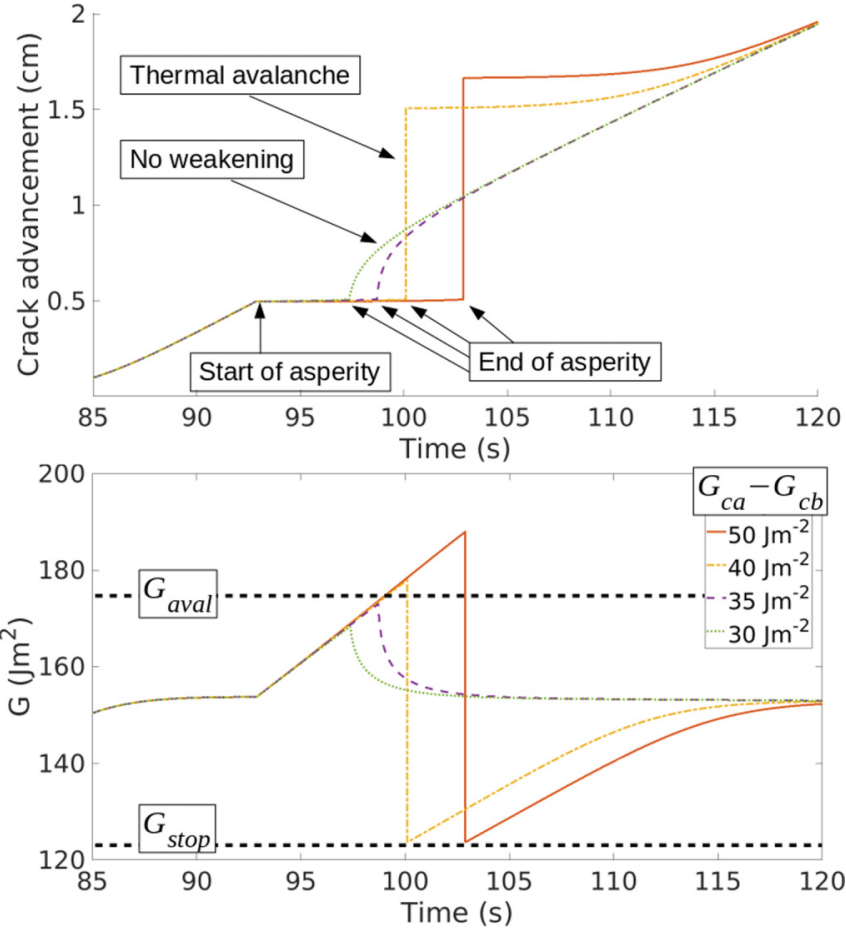

FIG. 6. Numerical simulations for a crack overcoming an asperity as defined by the differential equation from (3) and (10) and for various $G_{c_{a}} . L_{a}=100 \mu \mathrm{m}, v_{u}=120 \mu \mathrm{m} \mathrm{s}^{-1}, h=5 \mathrm{~mm}$, and $E=3.2 \mathrm{GPa}$. The top plot is the crack advancement $a(t)$, the bottom one is the energy release rate $G(t)$. Thermal weakening is or is not triggered depending on the anomaly strength.

\section{DISCUSSION AND CONCLUSION}

By combining an Arrhenius law and the heat equation, we have thus demonstrated the possibility of a thermally activated dynamic phase transition in the propagation of cracks. This phase description may have major implications for the understanding of fracture dynamics. With a rather simple subcritical model, we indeed explain both slow creep regimes and fast ruptures. We do not however strictly disregard over-critical propagations, as $G>G_{c}$ only implies that the Arrhenius activation energy is null and hence always exceeded. In this case, we predict $V \sim V_{0}$. Note that at such high velocities, crack fronts tend to complexify [36,37], and our model might not hold as such, as it only considers single fronts. We derive a tip temperature approaching the $10^{4} \mathrm{~K}$ range. Although it is high, some experimental characterizations of triboluminescense $[7,8]$ have shown that fast cracks can reach such a temperature, which only stands on small volumes $\left(\sim l^{2} L\right.$, where $L$ is the length of the front) and short time periods $(\sim l / V)$ such that it does not imply a gigantic level of energy nor it necessary leads to local fusion or sublimation of the solid. Note that the temperature merely measures the amplitude of the atoms agitation and its statistical definition actually suffers for heat production zones smaller than the molecular scale. While atomic scale simulations [38] would be more appropriate to study the induced heat, such computationally demanding models are often run at given (fixed) temperatures. Yet, some occurrences $[39,40]$ derive a non-negligible induced heat. 
Besides describing the two phases, we explained the potential shifts from one to the other and point out here how compatible this is with Maugis' reinterpretation [41] of the Griffith criteria [24] and so, with the usual stick-slip in brittle fracturing processes [20,30], when avalanches get considerably larger than the scales of the in situ quenched disorder. We also showed that above a critical ambient temperature, $T_{0}^{*}$, this phenomenon cannot occur. For materials where $T_{0}^{*}$ is lower than the melting point at a given confining pressure, the same solid then displays a different behavior under cool or hot conditions: fragile when cold, but smoother/ductile when warm, as thermal avalanches are inhibited. The model thus could stand as a novel and physical explanation for the fragile-ductile transition of matter. Of course, it might be oversimplifying to assume that all our parameters stay constant when varying $T_{0}$. The general physical principles, however, remain valid. Previous theories [42-44] actually support the importance of the crack-tip plasticity in the fragile-ductile transition, but rather relate it to the nucleation and mobility of dislocations ahead of the front. Such processes are compatible with induced thermal elevation [39], but are not directly captured by our mesoscopic description of the heat production zone.

Finally, and although we presented a mode I model, we suggest that some analogy is to be made with the frictional effects induced in mode II and mode III fracturing. Notably, as frictional heating is believed to be a cause for the instability of some seismic faults, a potential earthquake triggered when overcoming a strong fault plane asperity might indeed be amplified due to thermal weakening. The existence of the critical point would then explain the disappearance of such amplifications at higher depth (i.e., where rocks are in ductile conditions [45]) as the thermal background is there enough to make the frictional heating negligible and, hence, favors creep over brittle ruptures.

\section{ACKNOWLEDGMENTS}

T.V.-D. developed and analyzed the model and the simulations, and redacted the first versions of the manuscript. R.T. proposed the physical basis of the model and its mathematical formulation. A.C. set the basis for the numerical implementation of the model and the principles of the resolution algorithm. K.J.M. contributed in the interpretation of the model in fracture mechanics applications. E.G.F. contributed to analyze the model in terms of critical point characteristics. All authors participated to the redaction of the manuscript and agreed with the submitted version. The authors declare no competing financial interests in the publishing of this work and acknowledge the support of the IRP France-Norway DFFRACT, of the Universities of Strasbourg and Oslo and of the CNRS INSU ALEAS program. We thank the Research Council of Norway through its Centres of Excellence funding scheme, Project No. 262644.
[1] S. S. Brenner, Mechanical behavior of sapphire whiskers at elevated temperatures, J. Appl. Phys. 33, 33 (1962).

[2] S. N. Zhurkov, Kinetic concept of the strength of solids, Int. J. Fract. 26, 295 (1984).

[3] N. Brantut, M. J. Heap, P. G. Meredith, and P. Baud, Timedependent cracking and brittle creep in crustal rocks: A review, J. Struct. Geol. 52, 17 (2013).

[4] B. Lawn, Fracture of Brittle Solids, 2nd ed., Cambridge Solid State Science Series (Cambridge University Press, Cambridge, UK, 1993).

[5] J. R. Rice and N. Levy, Local heating by plastic deformation at a crack tip, in Physics of Strength and Plasticity, edited by A. S. Argon (MIT Press, Cambridge, MA, 1969), pp. 277-293.

[6] K. N. G. Fuller, P. G. Fox, and J. E. Field, The temperature rise at the tip of fast-moving cracks in glassy polymers, Proc. R. Soc. London A 341, 537 (1975).

[7] G. N. Chapman and A. J. Walton, Triboluminescence of glasses and quartz, J. Appl. Phys. 54, 5961 (1983).

[8] G. Pallares, C. L. Rountree, L. Douillard, F. Charra, and E. Bouchaud, Fractoluminescence characterization of the energy dissipated during fast fracture of glass, Europhys. Lett. 99, 28003 (2012).

[9] R. Toussaint, O. Lengliné, S. Santucci, T. Vincent-Dospital, M. Naert-Guillot, and K. J. Måløy, How cracks are hot and cool: a burning issue for paper, Soft Matter 12, 5563 (2016).

[10] S. Braeck and Y. Y. Podladchikov, Spontaneous Thermal Runaway as An Ultimate Failure Mechanism of Materials, Phys. Rev. Lett. 98, 095504 (2007).
[11] J. R. Rice, Heating and weakening of faults during earthquake slip, J. Geophys. Res. 111, B05311 (2006).

[12] H. Noda, E. M. Dunham, and J. R. Rice, Earthquake ruptures with thermal weakening and the operation of major faults at low overall stress levels, J. Geophys. Res. 114, B07302 (2009).

[13] G. P. Marshall, L. H. Coutts, and J. G. Williams, Temperature effects in the fracture of PMMA, J. Mater. Sci. 9, 1409 (1974).

[14] G. Carbone and B. N. J. Persson, Hot Cracks in Rubber: Origin of the Giant Toughness of Rubberlike Materials, Phys. Rev. Lett. 95, 114301 (2005).

[15] A. W. Rempel and J. R. Rice, Thermal pressurization and onset of melting in fault zones, J. Geophys. Res. 111, B09314 (2006).

[16] C. Wibberley and T. Shimamoto, Earthquake slip weakening and asperities explained by fluid pressurization, Nature (London) 436, 689 (2005).

[17] J. Sulem and V. Famin, Thermal decomposition of carbonates in fault zones: Slip-weakening and temperature-limiting effects, J. Geophys. Res. 114, B03309 (2009).

[18] G. G. Hammes, Principles of Chemical Kinetics (Academic Press, Cambridge, MA, 1978).

[19] T. Vincent-Dospital, R. Toussaint, S. Santucci, L. Vanel, D. Bonamy, L. Hattali, A. Cochard, K. J. Måløy, and E. G. Flekkøy, How heat controls fracture, Phys. Rev. Mater. (to be published).

[20] S. Santucci, L. Vanel, and S. Ciliberto, Subcritical Statistics in Rupture of Fibrous Materials: Experiments and Model, Phys. Rev. Lett. 93, 095505 (2004). 
[21] O. Lengliné, R. Toussaint, J. Schmittbuhl, J. E. Elkhoury, J. P. Ampuero, K. T. Tallakstad, S. Santucci, and K. J. Måløy, Average crack-front velocity during subcritical fracture propagation in a heterogeneous medium, Phys. Rev. E 84, 036104 (2011).

[22] K. T. Tallakstad, R. Toussaint, S. Santucci, J. Schmittbuhl, and K. J. Måløy, Local dynamics of a randomly pinned crack front during creep and forced propagation: An experimental study, Phys. Rev. E 83, 046108 (2011).

[23] A. Cochard, O. Lengliné, K. J. Måløy, and R. Toussaint, Thermally activated crack fronts propagating in pinning disorder: Simultaneous brittle/creep behavior depending on scale, Philos. Trans. R. Soc. A 377, 20170399 (2019).

[24] A. Griffith, The Phenomena of Rupture and Flow in Solids, Philos. Trans. R. Soc. London A 221, 163 (1921).

[25] L. B. Freund, Crack propagation in an elastic solid subjected to general loading, J. Mech. Phys. Solids 20, 129 (1972).

[26] G. R. Irwin, Analysis of stresses and strains near the end of a crack traversing a plate, J. Appl. Mech. 24, 361 (1957).

[27] See Supplemental Material at http://link.aps.org/supplemental/ 10.1103/PhysRevMaterials.4.023604 for a discussion on the parameters we used, details on how to compute the temperature elevation at the crack tip and decades on which we fitted the critical exponents

[28] D. K. Arrowsmith and C. M. Place, An Introduction to Dynamical Systems (Cambridge University Press, Cambridge, 1990).

[29] H. B. Callen, Thermodynamics and an Introduction to Thermostatistics, 2nd ed. (Wiley, New York, 1985).

[30] M.-J. Dalbe, S. Santucci, P.-P. Cortet, and L. Vanel, Strong dynamical effects during stick-slip adhesive peeling, Soft Matter 10, 132 (2014).

[31] M. Barquins and M. Ciccotti, On the kinetics of peeling of an adhesive tape under a constant imposed load, Int. J. Adhes. Adhes. 17, 65 (1997).

[32] M. L. Hattali, J. Barés, L. Ponson, and D. Bonamy, Low velocity surface fracture patterns in brittle material: A newly evidenced mechanical instability, in THERMEC 2011,
Volume 706 of Materials Science Forum (Trans Tech, 2012), pp. 920-924.

[33] Y. Morishita, K. Tsunoda, and K. Urayama, Velocity transition in the crack growth dynamics of filled elastomers: Contributions of nonlinear viscoelasticity, Phys. Rev. E 93, 043001 (2016).

[34] T. L. Anderson, Fracture Mechanics: Fundamentals and Applications (CRC Press, Boca Raton, FL, 2005).

[35] J. R. Dormand and P. J. Prince, A family of embedded RungeKutta formulae, J. Comput. Appl. Math. 6, 19 (1980).

[36] K. Ravi-Chandar and B. Yang, On the role of microcracks in the dynamic fracture of brittle materials, J. Mech. Phys. Solids 45, 535 (1997).

[37] J. Fineberg, S. P. Gross, M. Marder, and H. L. Swinney, Instability in Dynamic Fracture, Phys. Rev. Lett. 67, 457 (1991).

[38] C. L. Rountree, R. K. Kalia, E. Lidorikis, A. Nakano, L. Van Brutzel, and P. Vashishta, Atomistic aspects of crack propagation in brittle materials: Multimillion atom molecular dynamics simulations, Annu. Rev. Mater. Res. 32, 377 (2002).

[39] T. C. Germann, B. L. Holian, P. S. Lomdahl, D. Tanguy, M. Mareschal, and R. Ravelo, Dislocation structure behind a shock front in fcc perfect crystals: Atomistic simulation results, Metall. Mater. Trans. A 35, 2609 (2004).

[40] P. R. Budarapu, B. Javvaji, V. K. Sutrakar, D. Roy Mahapatra, G. Zi, and T. Rabczuk, Crack propagation in graphene, J. Appl. Phys. 118, 064307 (2015).

[41] D. Maugis, Subcritical crack growth, surface energy, fracture toughness, stick-slip and embrittlement, J. Mater. Sci. 20, 3041 (1985).

[42] P. B. Hirsch, S. G. Roberts, and J. Samuels, The brittle-ductile transition in silicon, Proc. R. Soc. London A 421, 25 (1989).

[43] D. Tanguy, Constrained molecular dynamics for quantifying intrinsic ductility versus brittleness, Phys. Rev. B 76, 144115 (2007).

[44] A. Hartmaier and P. Gumbsch, Thermal activation of crack-tip plasticity: The brittle or ductile response of a stationary crack loaded to failure, Phys. Rev. B 71, 024108 (2005).

[45] C. H. Scholz, The brittle-plastic transition and the depth of seismic faulting, Geologische Rundschau 77, 319 (1988). 